\title{
A CHARACTERIZATION OF CHAOS
}

\author{
K. Jankoyá and J. Smítal
}

Consider the continuous mappings $f$ from a compact real interval to itself. We show that when $f$ has a positive topological entropy (or equivalently, when $f$ has a cycle of order $\left.\neq 2^{n}, n=0,1,2, \ldots\right)$ then $f$ has a more complex behaviour than chaoticity in the sense of $\mathrm{Li}$ and Yorke: something like strong or uniform chaoticity, distinguishable on a certain level $\varepsilon>0$. Recent results of the second author then imply that any continuous map has exactly one of the following properties: It is either strongly chaotic or every trajectory is approximable by cycles. Also some other conditions characterizing chaos are given.

Denote by $C^{\rho}(I, I)$ the class of continuous mappings $I \rightarrow I$, where $I$ is a compact real interval. An $f \in C^{O}(I, I)$ is said to be chaotic in the sense of $\mathrm{Li}$ and Yorke [5], when there is an uncountable set $S \subseteq I$ such that for any $x, y \in S, x \neq y$, and any periodic point $p$ of $f$,

$$
\lim _{n \rightarrow \infty}\left|f^{n}(x)-f^{n}(y)\right|>0
$$

Received 25 November 1985.

Copyright Clearance Centre, Inc. Serial-fee code: 0004-9727/86 $\$ A 2.00+0.00$. 


$$
\lim _{n \rightarrow \infty}\left|f^{n}(x)-f^{n}(y)\right|=0
$$

$$
\lim _{n \rightarrow \infty} \sup _{n}\left|f^{n}(x)-f^{n}(\mathrm{p})\right|>0
$$

Here $f^{n}$ denotes the $n$-th iterate of $f$. Any set $S$ whose points satisfy condition (1) - (3) is called a scrambled set for $f$.

In [10] is given the following stronger concept: given $\varepsilon>0$, a set $S \subseteq I$ is an $\varepsilon$-scrambled set for some $f \in C^{O}(I, I)$ if for any $x, y \in S, x \neq y$, and any periodic point $p$ of $f$,

$$
\begin{aligned}
& \limsup _{n \rightarrow \infty}\left|f^{n}(x)-f^{n}(y)\right|>\varepsilon \\
& \limsup _{n \rightarrow \infty}\left|f^{n}(x)-f^{n}(p)\right|>\varepsilon
\end{aligned}
$$

and (2) is true.

Moreover, in [10] it is shown that for any $f \in C^{O}(I, I)$ with zero topological entropy (or equivalently, without cycles of order divisible by an odd prime, see [6]) the chaoticity in the sense of $\mathrm{Li}$ and Yorke is equivalent to the existence of a perfect non-empty $\varepsilon$-scrambled set, for some $E>0$. The following main result of this paper shows that this is also true for mappings with positive topological entropy.

THEOREM 1. Let $f \in C^{O}(I, I)$ have a cycle of order divisible by an odd prime. Then for some $\varepsilon>0, f$ has a non-empty perfect $\varepsilon-$ scrambled set $S$.

In the proof we use methods of symbolic dynamics, see, for example, [2] or [7]. First we recall the following well-known result.

LEMMA 1. (Block [1], see also [12]). If $f \in C^{O}(I, I)$ has a cycle of order $\neq 2^{n}, n=0,1,2, \ldots$, then there are closed disjoint intervals $J_{O}, J_{1} \subseteq I$ and an integer $m>0$ such that

$$
f^{m}\left(J_{0}\right) \cap f^{m}\left(J_{1}\right) \geq J_{0} \cup J_{1}
$$

Next we give a generalization of this lemma. 
LEMMA 2. Let $f, J_{\sigma}, J_{1}$ and $m$ be as in Lemma 1. Then there are closed intervals

$$
J_{0}=J_{0}^{0} \geq J_{1}^{0} \geq J_{2}^{0} \geq \cdots \quad \text { and } \quad J_{1}=J_{0}^{1} \geq J_{1}^{1} \geq J_{2}^{1} \geq \ldots
$$

and a sequence $\{m(k)\}_{k=0}^{\infty}$ of positive integers such that for every $k=0,1,2, \ldots$ and $j=0,1$,

$$
\begin{aligned}
& m(k) \text { is divisible by } k ! \text { and } m, \\
& f^{m(k)}\left(J_{k}^{j}\right) \geq J_{0} \cup J_{1}, \\
& \mu\left(J_{k+1}^{j}\right)<\frac{1}{2} \mu\left(J_{k}^{j}\right),
\end{aligned}
$$

where $\mu$ is the Lebesgue measure.

Proof. Put $m(0)=m$ and assume by induction that $m(k), J_{k}^{0}$ and $J_{k}^{1}$ are defined for $k \leq n$. Choose a closed interval $U_{j} \subseteq J_{n}^{0}$ such that $f^{m(n)}\left(U_{j}\right)=J_{j}$, for $j=0,1$. Then at least one of the sets $U_{0}, U_{1}$ has Lebesgue measure less than $\frac{1}{2} \mu\left(J_{n}^{0}\right)$. Denote this set by $J_{n+1}^{0}$ and put $m(n+1)=m(n)+p$, where $p \geq m$ is choosen such that (7) is true for $k=n+1$. Then by (6)

$$
f^{m(n+1)}\left(J_{n+1}^{0}\right)=f^{m(n)+p}\left(J_{n+1}^{0}\right)=f^{p}\left(J_{0}\right) \geq J_{0} \cup J_{1}
$$

since $p$ is divisible by $m$. Similarly we find $J_{n+1}^{1}$.

In the sequel the following notation will be useful. Let $X(k)$ be the set $\{0,1\}^{k}$ of all ordered $k$-tuples and $X=\{0,1\}^{N}$ the set of all sequences of two symbols 0,1 . If $\alpha \in X(k), \beta \in X(s)$ then $\alpha \beta \in X(k+s)$ is the concatenation of $\alpha$ and $\beta$. For $\alpha \in X(k)$ or $\alpha \in X, \alpha(j)$ will denote the $j$-th coordinate of $\alpha$. Assume $X$ is equipped with the topology of pointwise convergence (given for example by the metric $\left.\rho(\alpha, \beta)=\sum_{n} 2^{-n}|\alpha(n)-\beta(n)|\right)$.

LEMMA 3. There is a perfect, non-empty set $Y \subseteq X$ such that ony 
$\alpha \in Y$ has infinitely mony $O^{\prime} s$ and $I^{\prime} s$, and for any two $\alpha, \beta \in Y$, $\alpha \neq B$ implies $\alpha(n) \neq B(n)$ for infinitely many $n$.

Proof. Let $\xi$ be an irrational number. Define $\tau:[0,1] \rightarrow X$ in the following way: For $t \in[0,1], \tau(t)=\{\alpha(k)\}_{k=1}^{\infty}$, where

$$
\alpha(k)=\left\{\begin{array}{lll}
0 & \text { if } & N(t+\xi k) \in[0,1 / 2) \\
1 & \text { if } & N(t+\xi k) \in[1 / 2,1)
\end{array}\right.
$$

Here $N(x) \in[0,1)$ is the fractional part of $x$. Considering the wellknown fact that $\{N(\xi k)\}_{k=1}^{\infty}$ is uniformly distributed and hence dense in $[0,1]$, we can easily verify that $\tau(t)(n) \neq \tau(s)(n)$ for infinitely many $n$, whenever $t, s \in[0,1], t \neq s$.

Next observe that $\tau$ has at most a countable set of discontinuity points: for each $k$ there is exactly one $t \in[0,1]$ so that $N(t+\xi k)=1 / 2$. Denote this $t$ by $t(k)$. Clearly $\tau$ is continuous on $B=[0,1] \backslash\{t(k)\}_{k=1}^{\infty}$. Since $B$ is a Borel set we have that $\tau(B) \subseteq X$ is analytic and uncountable and by [4] it contains a non-empty perfect set $P$.

For any $\alpha \in P$ write

$$
\alpha^{*}=\alpha(1) \quad 0 \quad \alpha(2) \quad 1 \quad \alpha(3) \quad 0 \quad \alpha(4) \quad 1 \quad \alpha(5) \quad 0 \quad \ldots
$$

and let $Y=\left\{\alpha^{*} ; \alpha \in P\right\}$. It is easy to see that $Y$ is closed (as the intersection of closed sets) and has no isolated points, that is $Y$ is perfect.

LEMMA 4. Let $f$ have a cycle of order divisible by an odd prime. Then there is a set $\left\{I_{\alpha} ; \alpha \in X(k)\right\}_{k=1}^{\infty}$ of closed intervals and a sequence $\{n(k)\}_{k=1}^{\infty}$ of positive integers such that, for every $k, s$, $k>s$,

$$
\begin{aligned}
& I_{\alpha \beta} \subseteq I_{\alpha} \text { for } \quad \alpha \in X(k-s), \beta \in X(s), \\
& f^{n(k)}\left(I_{\alpha}\right)=J_{k}^{\alpha(k)} \text { whenever } \alpha \in X(k), \\
& n(k)-n(s) \text { is divisible by } s ! ;
\end{aligned}
$$


here $J_{k}^{i}$ are the intervals from Lemma 2.

Proof. Keep the notation from Lemma 2. Since $f^{m(0)}\left(J_{0}^{j}\right) \geq J_{0} \cup J_{1}$, there is a closed interval $I_{j} \subseteq J_{0}^{j}=J_{j}$ such that $f^{m(0)}\left(I_{j}\right)=J_{j}$, $j=0,1$. Put $n(1)=m(0)$.

Now assume by induction that we have defined intervals $\left\{I_{\alpha} ; \alpha \in X(r)\right\}$ and $n(r)$. Let $\alpha \in X(r+1)$. Then $\alpha=\beta O$ or $\alpha=\beta 1$ where $\beta \in X(r)$. By the hypothesis, $f^{n(r)}\left(I_{\beta}\right)=J_{r}^{\beta(r)}$ and Lemma 2 gives

$$
f^{n(r)+m(r)}\left(I_{B}\right) \geq I_{0} \cup I_{1}
$$

Hence there is a closed interval $I_{\alpha} \subseteq I_{\beta}$ such that $f^{n(r)+m(r)}\left(I_{\alpha}\right)=$ $J_{p+1}^{\alpha(r+1)}$. If we take $n(r+1)=n(r)+m(r)$, then by (7) and hypothesis, (12) if true for $k=r+1$. The other conditions are clearly satisfied. Now we are ready to give

Proof of Theorem 1. Keep the notation from Lemmas $1-4$. Write $F_{k}=U\left\{I_{\alpha} ; \alpha \in X(k)\right\}$ and $A=\prod_{k=1}^{\infty} F_{k}$. Define a mapping $\phi: A \rightarrow X$ in the following way:

For any $x \in A$ let $\phi(x)=\alpha \in X$ be such that $x \in M_{\alpha}$, where

$$
M_{\alpha}=I_{\alpha(1)} \cap I_{\alpha(1) \alpha(2)} \cap I_{\alpha(1) \alpha(2) \alpha(3)^{n}} \ldots
$$

(it is easy to see that for every $x$ there is exactly one $\alpha$ with $\left.x \in M_{\alpha}\right)$, since $M_{\alpha} \neq \varnothing$ for every $\alpha, \phi$ is surjective.

The mapping $\phi$ is also continuous. Indeed, let $O(\alpha)$ be a neighbourhood of $\alpha \in X$. Then there is an $n$ such that

$$
O(\alpha) \geq O^{n}(\alpha)=\{\beta \in X ; \beta(k)=\alpha(k) \text { for } k=1, \ldots, n\} .
$$

Write $G=I_{\alpha(1) \ldots \alpha(n)}$. Let $x \in A$ with $\phi(x)=\alpha$. Then $G$ is a relatively open neighbourhood of $x$ in $A$, and clearly $\phi(G) \subseteq O(\alpha)$.

Note that for every $\alpha, M_{\alpha}$ is closed and connected, and $\phi$ is constant on $M_{\alpha}$. Let $x_{\alpha}$ be the left-end point of $M_{\alpha}$. Then clearly 
$B=\left\{x_{\alpha} ; \alpha \in X\right\} \subseteq A$ is an uncountable Borel set and $\phi$ restricted to $B$ is a bijection $B \rightarrow A$. Therefore $\phi^{-1}(Y) \cap B$ is an uncountable Borel set. Hence there is a non-empty perfect set $S \subseteq \phi^{-1}(Y) \cap B$ (see [4]; here $Y$ is the set from Lemma 3 ).

It remains to verify that $S$ is the desired $\varepsilon$-scrambled set for $f$, where

$$
\varepsilon=\frac{1}{3} \operatorname{dist}\left(J_{0}, J_{1}\right)>0
$$

Let $x, y \in S, x \neq y$. Then $\phi(x)=\alpha, \phi(y)=\beta$, where $\alpha \neq \beta$, $\alpha, \beta \in Y$. Hence by Lemma 3 and (11), for infinitely many $k$ either

$$
f^{n(k)}(x) \in J_{0} \quad \text { and } \quad f^{n(k)}(y) \in J_{1}
$$

or

$$
f^{n(k)}(x) \in J_{1} \quad \text { and } \quad f^{n(k)}(y) \in J_{0}
$$

since $J_{j}^{i} \subseteq J_{i}$ for every $i, j$. Thus (4) is true.

Again by Lemma 3 and (11), for infinitely many $k$ we have

$\alpha(k)=\beta(k)$, and thus $f^{n(k)}(x), f^{n(k)}(y) \in J_{k}^{\alpha(k)}=J_{k}^{\beta(k)}$, hence by (9)

$$
\left|f^{n(k)}(x)-f^{n(k)}(y)\right| \leq \mu\left(J_{k}^{\alpha(k)}\right) \leq 2^{-k} \mu\left(J_{0}^{\alpha(k)}\right)
$$

for every such $k$ and this implies (2).

Finally, let $x \in S$ and let $p \in I$ be a periodic point of $f$. Let $s$ be the period of $p$. For $k>s$ we have

$$
f^{n(k)}(p)=f^{n(k)-n(s)}\left(f^{n(s)}(p)\right)=f^{n(k)-n(s)}(q)=q .
$$

since $q$ has period $s$ and $s$ divides $n(k)-n(s)$ (see (12)). Let $r \in\{0,1\}$ be such that dist $\left(J_{r},\{q\}\right)>\varepsilon$. Choose $k>s$ so that for $\alpha=\phi(x), \alpha(k)=r$. Then by (1I),

$$
\left|f^{n(k)}(x)-f^{n(k)}(p)\right| \geq \operatorname{dist}\left(J_{p},\{q\}\right)>\varepsilon
$$

Since $k$ can be choosen arbitrarily large we obtain (5) and our theorem is proved.

Before we state the next result, we recall some terminology (see 
[10]). Let $f \in C^{0}(I, I)$. We say that an interval $J \subseteq I$ is an $f$ periodic interval of order $k$ if $f^{k}(J)=J$ and $f^{i}(J) \cap f^{j}(J)=\emptyset$ for $i \neq j, i, j=1, \ldots, k$. Two points $u, v \in I$ are $f$-separable if there are disjoint periodic intervals $J_{u}, c_{v}^{T} \subseteq I$ with $u \in J_{u}, v \in J_{v}$. otherwise $u, v$ are $f$-nonseparable. The set of all limit points of a trajectory $\left\{f^{k}(x)\right\}_{k=1}^{\infty}$ is called the attractor of $f$ and $x$, and is denoted by $L_{f}(x)$.

The following theorem generalizes a result from [10].

THEOREM 2. A function $f \in C^{O}(I, I)$ is chaotic in the sense of $L i$ and Yorke if and only if there is an infinite attractor $L_{f}(x)$ containing two f-nonseparable points $u, v$.

Proof. In [10] the theorem is proved for functions with zero topological entropy. Thus in view of Theorem 1 it suffices to show that any $f \in C^{O}(I, I)$ with positive topological entropy has an infinite attractor $L_{f}(x)$ containing two $f$-nonseparable points $u, v$.

Hence assume $f$ has a cycle of order divisible by an odd prime (see [6]). By [11] or [12] there is an uncountable attractor $L_{f}(x)$ containing a cycle of $f$. Let the order of this cycle be $m \geq 1$. clearly $L_{f}(x)$ contains two accumulation points $u, v$ of $L_{f}(x)$. Assume that there are disjoint periodic intervals $J_{u}, J_{v}, u \in J_{u}, v \in J_{v}$, with periods $m(u), m(v) \geq 1$ (otherwise $u$ and $v$ would be $f$-nonseparable).

Then there is a $k$ such that $f^{k}(x) \in J_{u}$, and hence $L_{f}(x) \subseteq$ $\operatorname{Orb}_{f}\left(J_{u}\right)=\sum_{i=1}^{m(u)} f^{i}\left(J_{u}\right)$, and similarly $L_{f}(x) \subseteq \operatorname{Orb}_{f}\left(J_{v}\right)$. since $J_{u}, J_{v}$ are disjoint, we have $m(u)>1, m(v)>1$. Consider the mapping $f^{m(n)}$ restricted to $J_{u}$; denote it $f_{1}$. By the periodicity of $J_{u}$, the set $L_{f}(x) \cap J_{u}$ is uncountable (since $f\left(L_{f}(x)\right)=L_{f}(x)$ ). Choose two accumulation points $u_{1}, v_{1} \in L_{f}(x) \cap J_{u}$ of $L_{f}(x)$. Assume there 
are disjoint $f_{1}$-periodic intervals $J_{u}^{1}, J_{v}^{1} \subset J_{u}$ with periods $m\left(u_{1}\right)$, $m\left(v_{1}\right) \geq 1$ such that $u_{1} \in J_{u}^{1}$ and $v_{1} \in J_{v}^{1}$ (otherwise $u_{1}, v_{1}$ are $f$ nonseparable). Similarly as in the preceding step we can see that $m\left(u_{1}\right)>1$ and $m\left(v_{1}\right)>1$. Hence $\delta_{u}^{1}$ is an $f$-periodic interval of period $m(u) \cdot m\left(u_{1}\right)>m(u)$, and such that $L_{f}(x) \subseteq \operatorname{orb}_{f}\left(J_{u}^{1}\right)$.

By repeating this construction we obtain $f$-periodic intervals $J_{u} \geq J_{u}^{1} \supseteq J_{u}^{2} \supseteq \cdots \geq J_{u}^{n}$, where $n \geq 1$ is the first index such that $L_{f}(x) \subseteq \operatorname{orb}_{f}\left(J_{u}^{n}\right)$ and $m\left(u_{n}\right)$, the period of $J_{u}^{n}$, is greater than $m$. But this is a contradiction with the fact that $L_{f}(x)$ contains a cycle of order $m$. Hence $u_{n-1}, v_{n-1}$ are $f$-nonseparable.

Now we can prove the following survey theorem summarizing conditions equivalent to the chaoticity of mappings. Recall that for $f \in c(I, I)$ we say that the trajectory $\left\{f^{k}(x)\right\}_{k=1}^{\infty}$ of $x$ is approximable by cycles if for any $\varepsilon>0$ there is a pexiodic point $p$ of $f$ such that

$$
\lim _{n \rightarrow \infty} \sup _{n}\left|f^{n}(x)-f^{n}(p)\right|<\varepsilon \text {. }
$$

THEOREM 3. Let $f \in C^{O}(I, I)$. The following conditions are equivalent:

(a) $f$ is chaotic in the sense of Li cond Yorke;

(b) $f$ has con infinite attractor containing two f-nonseparable points;

(c) for some $\varepsilon>0, f$ has a nonempty perfect $\varepsilon$-scrambled set;

(d) $f$ has a trajectory which is not approximable by cycles;

(e) $f$ is topologically conjugate to a function which has a scrambled set of positive Lebesgue measure;

(f) for some $\varepsilon>0, f$ has a nonempty $\varepsilon$-scrambled set.

Remark 1. We emphasize that, rather surprisingly, positive topological entropy (or the existence of a cycle of order divisible by an odd prime) is not equivalent to the chaoticity of a function $f$ in 
the sense of $\mathrm{Li}$ and Yorke (an example is given in [10]). However, in view of Theorem 1, positive topological entropy of $f$ implies that chaoticity of $f$.

On the other hand, existence of an infinite attractor does not imply (but is clearly implied by) the chaoticity of $f$, see [10].

Remark 2. The implication $(a) \rightarrow(e)$ in Theorem 3 generalizes recent results [3], [7], [8], in which particular functions with large (from a measure-theoretical point of view) scrambled sets are constructed. However, this implication does not generalize the result from [9], in which map $g$ with a perfect scrambled set of positive Lebesgue measure is given. This is because $g$ can easily be modified to be of class $C^{I}$ (this possibility is not mentioned in [9]).

Proof of Theorem 3. (a) $\leftrightarrow$ (b): This follows from Theorem 2. $(b) \rightarrow(c)$ : This was proved in [10] for functions having no cycles of order divisible by an odd prime; for other functions use Theorem 1 .

$(c) \rightarrow(d)$ : This follows immediately from (5).

$(d) \rightarrow(a \vee b)$ : For functions with zero topological entropy the implication $(d) \rightarrow(b)$ is proved in [10], otherwise Theorem 1 gives the validity of (a).

(c) $\rightarrow(e)$ : Let $S \neq \emptyset$ be a perfect scrambled set for $f$. Let $h: I \rightarrow I$ be a homeomorphism such that $\mu(h(S))>0$. Then $h(S)$ is clearly a scrambled set for $g=h \circ f \circ h^{-1}$ (first apply $h^{-1}$ ).

$(e) \rightarrow(a)$ is trivial and since (f) is an another formulation of (d), also (d) $\leftrightarrow$ (f) is true.

Problem. It is possible to show that for $f \in C^{O}(I, I)$ the following condition also is equivalent to the chaoticity of $f$ :

(g) $f$ has a scrambled set containing two points. However, our proof is rather complicated. But this result should be probably provable in a simpler way. (Clearly, in view of Theorem 1 it suffices to consider only mappings with zero topological entropy satisfying the condition $(g)$ ) 


\section{References}

[1] L. Block, "Homoclinic mappings of the interval", Proc. Amer. Math. Soc. 72 (1978), 576-580.

[2] P. Collet and J. -P. Eckmann, Iterated maps of the interval as dynamical systems, Progress in Physics 1 (Birkhauser, 1980).

[3] I. Kan, "A chaotic function possessing a scrambled set of positive Lebesgue measure", Proc. Amer. Math. Soc. 92 (1984), 45-49.

[4] K. Kuratowski, Topologie I, (PWN - Polish Scientific Publishers, Warsaw 1968), p. 387.

[5] T. Y. Li, J. A. Yorke, "Period three implies chaos", Amer. Math. Monthly 82 (1975), 985-992.

[6] M. Misiurewicz, "Horseshoes for mappings of the interval", Buzl. Acad. Polon. Sci. Sér. Sci. Math. 27 (1979), 167-169.

[1] M. Misiurewicz, "Chaos almost everywhere", Iteration Theory and its Functional Equations (editor Liedl et al.), Lecture notes in mathematics (Springer 1985).

[8] J. Smital, "A chaotic function with some extremal properties", Proc. Amer. Math. Soc. 87 (1983), 54-56.

[9] J. Smítal, "A chaotic function with a scrambled set of positive Lebesgue measure", Proc. Amer. Math. Soc. 92 (1984), 50-54.

[10] J. Smítal, "Chaotic functions with zero topological entropy", Trons. Amer. Math. Soc. (to appear).

[11] A. N. Sarkovskii, "Attracted and attracting sets" (Russian), DokZ. AN SSSR 160 (1965), 1036-1038 = (English) Soviet Math. DokZ. 6 (1965), 268-270.

[12] A. N. Sarkovskii,"Behavior of a mapping in the neighborhood of an attracting set" (Russian), Ukrain. Math. Zh. 18 (1966) No. 2, 60-83 = (English) AMS Trans. (2) 97 (1970), 227-258.

Department of Mathematics

Komensky University

84215 Bratislava

Czechoslovakia. 